\title{
ON A QUADRATIC DIOPHANTINE INEQUALITY
}

\author{
FRED SUPNICK
}

1. Introduction. Let $C$ be an $n$-cube and $S$ the $n$-sphere circumscribed about $C$. Keeping $C$ fixed let $S$ be moved so that its center falls at some point $P$ inside or on $C$. We pose the problem: How can vertices of $C$ falling inside or on $S_{P}$ (the subscript denotes the center) be selected?

Analytically expressed, let $C$ be the unit $n$-cube the coordinates of whose vertices are zeros or ones (on a Cartesian coordinate system in $\left.E_{n}\right)$. Let $P$ be the point $\left(x_{1}, \cdots, x_{n}\right)$ with $0 \leqq x_{i} \leqq 1(i=1, \cdots, n)$. $S_{P}$ is of diameter $n^{1 / 2}$. We seek lattice points $\left(y_{1}, \cdots, y_{n}\right) y_{i}=0$ or $1(i=1, \cdots, n)$ satisfying

$$
\sum_{i=1}^{n}\left(x_{i}-y_{i}\right)^{2} \leqq n / 4
$$

Thus, trivially, one point $\left(y_{1}, \cdots, y_{n}\right)$ may always be obtained if we let $y_{i}=0$ if $x_{i} \leqq 1 / 2$ and $y_{i}=1$ if $x_{i}>1 / 2$.

Of course, one obvious method would be to substitute (the coordinates of) the vertices of $C$ into (1.1) and to select those which satisfy it; however, except for small $n$ this is a prohibitive operation (even with mechanical aid). The problem therefore is one of minimizing the number of operations in obtaining solutions of the desired type.

In this paper we obtain a process for immediately associating with any $\left(x_{1}, \cdots, x_{n}\right)\left(0 \leqq x_{i} \leqq 1, i=1, \cdots, n, n \geqq 4\right)$ a class of lattice points $\left(y_{1}, \cdots, y_{n}\right) y_{i}=0$ or $1(i=1, \cdots, n)$ satisfying (1.1).

We note that a lemma to a theorem of $\mathrm{D}$. Warncke and the author ${ }^{1}$ establishes the following class of solutions for the case $n=4$ : Let $\left(x_{i_{1}}, \cdots, x_{i_{4}}\right)$ be a rearrangement $G$ of $\left(x_{1}, \cdots, x_{4}\right)$ for which

$$
\left|x_{i_{1}}-1 / 2\right| \leqq\left|x_{i_{2}}-1 / 2\right| \leqq\left|x_{i_{3}}-1 / 2\right| \leqq\left|x_{i_{4}}-1 / 2\right| \text {. }
$$

Let $y_{i_{1}}^{\prime}=0$ and $y_{i_{1}}^{\prime \prime}=1$, and

$$
y_{i_{j}}^{\prime}=y_{i_{j}}^{\prime \prime}=\begin{array}{ll}
0 & \text { if } x_{i_{j}} \leqq 1 / 2 \\
1 & \text { if } x_{i_{j}}>1 / 2
\end{array}
$$

for $j=2,3,4$. Applying $G^{-1}$ to $\left(y_{i_{1}}^{\prime}, \cdots, y_{i_{4}}^{\prime}\right)$ and $\left(y_{i_{1}}^{\prime \prime}, \cdots, y_{i_{4}}^{\prime \prime}\right)$ we obtain lattice points $\left(y_{1}^{\prime}, \cdots, y_{4}^{\prime}\right)$ and $\left(y_{1}^{\prime \prime}, \cdots, y_{4}^{\prime \prime}\right)$ respectively (with coordinates zeros or ones) satisfying (1.1).

Received by the editors September 2, 1959, and, in revised form, March 16, 1960.

1 D. Warncke and F. Supnick, On the covering of $E_{n}$ by spheres, Proc. Amer. Math. Soc. vol. 8 (1957) pp. 299-303, 1160. See $\$ 2$. 
2. Some definitions and statement of results. An ordered set of integers $\left(a_{1}, \cdots, a_{r}\right)\left(1 \leqq a_{1}<a_{2}<\cdots<a_{r}, 1 \leqq r \leqq[n / r], n \geqq 4\right)$ will be called a primary set of order $r$ if

$$
a_{i} \leqq(n-3)-4(r-i) \quad(i=1, \cdots, r) .
$$

Let $\left(x_{1}, \cdots, x_{n}\right)\left(0 \leqq x_{i} \leqq 1, i=1, \cdots, n ; n \geqq 4\right)$ be arbitrarily chosen (but held fixed in the following argument). Let $\left(x_{i_{1}}, \cdots, x_{i_{n}}\right)$ be a rearrangement $H$ of $\left(x_{1}, \cdots, x_{n}\right)$ for which

$$
\left|x_{i_{1}}-\frac{1}{2}\right| \leqq\left|x_{i_{2}}-\frac{1}{2}\right| \leqq \cdots \leqq\left|x_{i_{n}}-\frac{1}{2}\right| \text {. }
$$

Let $z_{1}, \cdots, z_{n}$ denote $x_{i_{1}}, \cdots, x_{i_{n}}$ respectively.

Now, each primary set $\left(a_{1}, \cdots, a_{r}\right)$ induces a partition ${ }^{2}$

$$
\{1, \cdots, n\}=F+N
$$

where

$$
F=\left\{a_{1}, \cdots, a_{r}\right\}, \quad N=\{1, \cdots, n\}-\left\{a_{1}, \cdots, a_{r}\right\} .
$$

Let $k$ range over $\{1, \cdots, n\}$ :

(i) if $k \in F$, let

$$
p_{k}=\begin{array}{ll}
0 & \text { if } z_{k}>1 / 2 \\
1 & \text { if } z_{k} \leqq 1 / 2
\end{array}
$$

(ii) if $k \in N$, let

$$
p_{k}=\begin{array}{ll}
0 & \text { if } z_{k} \leqq 1 / 2 ; \\
1 & \text { if } z_{k}>1 / 2 .
\end{array}
$$

Now, because of (2.1), with each element $a_{i}$ of $F$ (cf. (2.3)) may be associated integers $b_{i}, c_{i}, d_{i}$ of $N$ (cf. (2.3)) such that $a_{i}<b_{i}<c_{i}<d_{i}$, holds for $(i=1, \cdots, r)$, and such that (the intersection) $\left\{a_{j}, b_{j}, c_{j}, d_{j}\right\}$ $\cdot\left\{a_{k}, b_{k}, c_{k}, d_{k}\right\}$ is null for all pairs $j, k \in\{1, \cdots, r\}(j \neq k)$. Recalling the solutions for the case $n=4$ (at the end of $\$ 1$ ) we have,

$$
\left(z_{a_{i}}-p_{a_{i}}\right)^{2}+\left(z_{b_{i}}-p_{b_{i}}\right)^{2}+\left(z_{c_{i}}-p_{c_{i}}\right)^{2}+\left(z_{d_{i}}-p_{d_{i}}\right)^{2} \leqq 1
$$

for $(i=1, \cdots, r)$. We note that $\left(z_{i}-p_{i}\right)^{2} \leqq 1 / 4$ for each element $i$ of

$$
\{1, \cdots, n\}-\sum_{i=1}^{r}\left\{a_{i}, b_{i}, c_{i}, d_{i}\right\}
$$

\footnotetext{
${ }^{2}$ We use the symbol \{\} to denote "unordered set". The operations " + ", " -", "." between unordered sets are those in common usage in set theory.
} 
(if indeed there are such). Therefore $\sum_{k=1}^{n}\left(z_{k}-p_{k}\right)^{2} \leqq n / 4$. Applying $H^{-1}$ to $\left(p_{1}, \cdots, p_{n}\right)$ we obtain a lattice point $\left(y_{1}, \cdots, y_{n}\right)\left(y_{i}=0\right.$ or 1$)$ satisfying (1.1). We call $\left(y_{1}, \cdots, y_{n}\right)$ an $(H)$-lattice-point (since it depends on $H$ ) associated with the primary set $\left(a_{1}, \cdots, a_{r}\right)$. The lattice point obtained by letting $y_{i}=0$ if $x_{i} \leqq 1 / 2$ and $y_{i}=1$ if $x_{i}>1 / 2(i=1, \cdots, n)$ will be referred to as the $(H)$-lattice-point associated with the null set (which we here call the "primary set of order zero" for convenience of exposition).

Statement of Results. Let $A_{i, j}$ denote the element in the $i$ th row and $j$ th column of the double array:

$\begin{array}{llllllllllllllllll}1 & 1 & 1 & 1 & 1 & 1 & 1 & 1 & 1 & 1 & 1 & 1 & 1 & 1 & 1 & 1 & 1 & \ldots \\ 0 & 0 & 0 & 0 & 4 & 5 & 6 & 7 & 8 & 9 & 10 & 11 & 12 & 13 & 14 & 15 & 16 & \ldots \\ 0 & 0 & 0 & 0 & 0 & 0 & 0 & 0 & 22 & 30 & 39 & 49 & 60 & 72 & 85 & 99 & 114 & \ldots \\ 0 & 0 & 0 & 0 & 0 & 0 & 0 & 0 & 0 & 0 & 0 & 0 & 140 & 200 & 272 & 357 & 456 & \ldots \\ 0 & 0 & 0 & 0 & 0 & 0 & 0 & 0 & 0 & 0 & 0 & 0 & 0 & 0 & 0 & 0 & 969 & \ldots\end{array}$

i.e., (i) if $i=1$, then $A_{i, j}=1$ for all positive integers $j$, (ii) if $i>1$, then $A_{i, j}=0$ for each positive integer $j \leqq 4(i-1)$, and

$$
A_{i, j}=\sum_{k=4(i-2)+1}^{j-1} A_{i-1, k}
$$

for each positive integer $j>4(i-1)$.

First an algorithm is given (cf. $\$ 4$ ) for listing all primary sets of order $r(1 \leqq r \leqq[n / 4])$, and the following theorem concerning the "length" of a complete listing is established:

THEOREM 1. For a given $n \geqq 4$, the total number of primary sets of orders $0,1, \cdots,[n / 4]$ is

$$
\theta \equiv 1+\sum_{j=1}^{n-3} \sum_{i=1}^{[n / 4]} A_{i, j}
$$

THEOREM 2. (i) Each primary set (which may be the primary set of order zero) has one and only one associated $(H)$-lattice-point, and (ii) distinct primary sets have distinct associated $(H)$-lattice-points.

We thus have the following constructive process for obtaining vertices of $C$ inside or on $S_{P}$ :

STEP 1. Once $n \geqq 4$ is specified, list all $\theta$ primary sets. This may be done by the algorithm of $\$ 4$.

STEP 2. Once $\left(x_{1}, \cdots, x_{n}\right)$ is specified determine a rearrangement $H$ 
yielding $\left(z_{1}, \cdots, z_{n}\right)$. Fixing attention on each primary set in turn, apply (2.4) and (2.5) to $\left(z_{1}, \cdots, z_{n}\right)$, thus obtaining $\left(p_{1}, \cdots, p_{n}\right)$; we then apply $H^{-1}$ to $\left(p_{1}, \cdots, p_{n}\right)$ and obtain an $(H)$-lattice-point satisfying (1.1).

REMARK. If $\left(x_{1}, \cdots, x_{n}\right)$ is such that $\left|x_{i}-1 / 2\right| \neq\left|x_{j}-1 / 2\right|$ for all pairs $i, j(i \neq j)$, then there is only one rearrangement $H$ satisfying (2.2). If $\left(x_{1}, \cdots, x_{n}\right)$ is such that $\left|x_{i}-1 / 2\right|=\left|x_{j}-1 / 2\right|$ for some pair $i, j(i \neq j)$, let

$$
H_{1}:\left(x_{i_{1}}, \cdots, x_{i_{n}}\right), \quad H_{2}:\left(x_{j_{1}}, \cdots, x_{j_{n}}\right), \cdots
$$

be all the rearrangements of $\left(x_{1}, \cdots, x_{n}\right)$ such that

$$
\begin{aligned}
& \left|x_{i_{1}}-1 / 2\right| \leqq \cdots \leqq\left|x_{i_{n}}-1 / 2\right|, \\
& \left|x_{j_{1}}-1 / 2\right| \leqq \cdots \leqq\left|x_{j_{n}}-1 / 2\right|, \cdots .
\end{aligned}
$$

Let all $\theta\left(H_{1}\right)$-lattice-points be obtained. To find those $\left(H_{2}\right)$-latticepoints which are not $\left(H_{1}\right)$-lattice-points, we need only consider primary sets $\left(a_{1}, \cdots, a_{r}\right)$ such that $\left(j_{a_{1}}, \cdots, j_{a_{r}}\right) \not \equiv\left(i_{a_{1}}, \cdots, i_{a_{r}}\right)$. Bearing this in mind at all times, we may obtain all other lattice points associated with each element of (2.8) without duplications.

3. A lemma. Let $\Delta_{r}(n)(1 \leqq r \leqq[n / 4])$ denote the matrix in the upper left-hand corner of (2.6) consisting of all elements $A_{i, j}(i=1, \cdots, r$; $j=1, \cdots, n-3)$. It will be convenient to introduce a new designation for an arbitrary element of $\Delta_{r}(n)$, say $s_{i, k}$, where $i$ indicates the $i$ th row from the top (as before), but $k$ now indicates the $k$ th column from the right; (thus $A_{i, j}=s_{i, k}$ where $k=n-2-j(j=1, \cdots, n-3)$ ).

Lemma. Let $s_{i, k}(i>1)$ be any nonzero element of $\Delta_{m}(n)(m=[n / 4])$ such that $s_{i, k+1}$ is not zero. Then

$$
s_{i, k}=s_{i, k+1}+s_{i-1, k+2}+s_{i-2, k+3}+\cdots+1 .
$$

ProOF. From (2.7) it follows that $s_{i, k}=s_{i, k+1}+s_{i-1, k+1}$. Since $s_{i-1, k+1}=s_{i-1, k+2}+s_{i-2, k+2}$, we obtain

$$
s_{i, k}=s_{i, k+1}+s_{i-1, k+2}+s_{i-2, k+2} .
$$

Repeating this argument on the last term of (3.2), etc., we finally obtain (3.1).

4. An algorithm. Let $S_{r}$ denote the sum of the elements of the $r$ th row of $\Delta_{r}(n)(1 \leqq r \leqq[n / 4], n \geqq 4)$. Let $q_{0}$ be an integer satisfying $1 \leqq q_{0} \leqq S_{r}$. We shall associate with $q_{0}$ a primary set $\left(j_{0}, j_{1}, \cdots, j_{r-1}\right)$ as follows:

(1) Determination of $j_{0}$. We notice that 


$$
S_{r}=s_{r, v_{0}}+s_{r, v_{0}-1}+\cdots+s_{r, 2}+s_{r, 1}
$$

where $v_{0}=(n-3)-(r-1) 4$; there are $(r-1) 4$ zeros to the left of $s_{r, v_{0}}$ in the last row of $\Delta_{r}(n)$. Then from (4.1) we see that there is one and only one integer $j_{0}$ satisfying $1 \leqq j_{0} \leqq(n-3)-(r-1) 4$ such that

$$
\sum_{j=j_{0}+1}^{v_{0}} s_{r, j}<q_{0} \leqq \sum_{j=j_{0}}^{v_{0}} s_{r, j}
$$

(here and below, expressions of the form $\sum_{j-A}^{B} U_{j}$ where $B<A$ are to be taken as zero).

(2) Determination of $j_{1}$. We notice that

$$
s_{r, j_{0}}=s_{r-1, v_{1}}+s_{r-1, v_{1}-1}+\cdots+s_{r-1, j_{0}+1}
$$

where $v_{1}=(n-3)-(r-2) 4$; there are $(r-2) 4$ zeros to the left of $s_{r-1, v_{1}}$ in the $(r-1)$ st row of $\Delta_{r}(n)$. Let

$$
q_{1}=q_{0}-\sum_{j=j_{0}+1}^{v_{0}} s_{r, j}
$$

then $1 \leqq q_{1} \leqq s_{r, j_{0}}$. From (4.3) we see that there is one and only one integer $j_{1}$ satisfying $j_{0}<j_{1} \leqq(n-3)-(r-2) 4$ such that

$$
\sum_{j=j_{1}+1}^{v_{1}} s_{r-1, j}<q_{1} \leqq \sum_{j=j_{1}}^{v_{1}} s_{r-1, j}
$$

(3) Let us suppose that integers $j_{0}, j_{1}, \cdots, j_{i-1}$ have been determined, each $j_{o}(g \in\{1,2, \cdots, i-1\})$ being the only integer which satisfies

$$
j_{o-1}<j_{0} \leqq(n-3)-(r-(g+1)) 4 \equiv v_{0}
$$

and

$$
\sum_{j=j_{0+1}}^{v_{0}} s_{r-0, j}<q_{0} \leqq \sum_{j=j_{0}}^{v_{0}} s_{r-0, j}
$$

where

$$
q_{o}=q_{o-1}-\sum_{j-j_{0-1}+1}^{v_{g}-1} s_{r-(o-1), j}
$$

i.e.,

$$
q_{0}=q_{0}-\left(\sum_{j=j_{0}+1}^{v_{0}} s_{r, j}+\sum_{j=j_{1}+1}^{v_{1}} s_{r-1, j}+\cdots+\sum_{j=j_{0-1+1}}^{v_{0-1}} s_{r-(0-1), j}\right) .
$$


We show how to determine $j_{i}$. We notice that (if $r-(i-1) \geqq 2$ )

(4.7) $s_{r-(i-1), j_{i-1}}=s_{r-i, v_{i}}+s_{r-i,\left(v_{i}-1\right)}+\cdots+s_{r-i,\left(j_{i-1}+1\right)}$,

where $v_{i}=v_{0}+4 i$; there are $(r-(i+1)) 4$ zeros to the left of $s_{r-i, v_{i}}$ in the $(r-i)$ th row of $\Delta_{r}(n)$. Let

$$
q_{i}=q_{i-1}-\sum_{j=j_{i-1}+1}^{v_{i-1}} s_{r-(i-1), j}
$$

Then, letting $g=i-1$ in (4.6), and subtracting the left sum, we obtain $1 \leqq q_{i} \leqq s_{r-(i-1), j_{i-1}}$. From (4.7) we see that there is one and only one integer $j_{i}$ satisfying

$$
j_{i-1}<j_{i} \leqq(n-3)-(r-(i+1)) 4 \equiv v_{i}
$$

such that

$$
\sum_{j=j_{i}+1}^{v_{i}} s_{r-i, j}<q_{i} \leqq \sum_{j=j_{i}}^{v_{i}} s_{r-i, j}
$$

Repeatedly selecting the $j_{i}$ as described in (3) (immediately above), we finally obtain the primary set $\left(j_{0}, \cdots, j_{r-1}\right)$ which we associate with $q_{0}$.

From the manner in which $\left(j_{0}, \cdots, j_{r-1}\right)$ was selected we may now show that

$$
q_{0}=1+\sum_{k=0}^{r-1} \sum_{j=j_{k}+1}^{v_{k}} s_{r-k, j}
$$

Proof of (4.11). Let

$$
\beta_{k}=\sum_{j=j_{k}+1}^{v_{k}} s_{r-k, j}
$$

Then from (4.8) $q_{0}=q_{1}+\beta_{0}, q_{1}=q_{2}+\beta_{1}, \cdots$; therefore

$$
q_{0}=q_{r-1}+\beta_{r-2}+\beta_{r-3}+\cdots+\beta_{0} .
$$

But from (4.10), since $s_{1, j}=1$

$$
q_{r-1}=\sum_{j-j r_{-1}}^{v_{r-1}} s_{1, j}=1+\beta_{r-1},
$$

and (4.13) becomes

$$
q_{0}=1+\sum_{k=0}^{r-1} \beta_{k} .
$$

Substituting (4.12) into (4.14) we obtain (4.11). 
5. The number of primary sets $\left(a_{1}, \cdots, a_{r}\right)$. With any integer $g_{0}\left(1 \leqq g_{0} \leqq S_{r}\right)$ we have associated a primary set $\left(j_{0}, j_{1}, \cdots, j_{r-1}\right)$ determined as in $\$ 4$.

We now show that any primary set $\left(h_{0}, h_{1}, \cdots, h_{r-1}\right)$ is an associate of an integer $I$ satisfying $1 \leqq I \leqq S_{r}$.

(A) Let

$$
I \equiv 1+\sum_{k=0}^{r-1} \sum_{j=h_{k}+1}^{v_{k}} s_{r-k, j} .
$$

It is clear that $I \geqq 1$. We first prove that

$$
I \leqq S_{r} \text {. }
$$

Proof of (5.2). (i) Suppose there are at least two nonzero terms in the last row of $\Delta_{r}(n)$. Then

$$
\begin{aligned}
1+\sum_{k=0}^{r-1} \sum_{j=h_{k}+1}^{v_{k}} s_{r-k, j} & \leqq 1+\sum_{k=0}^{r-1} \sum_{j=k+2}^{v_{k}} s_{r-k, j} \\
& =1+\sum_{k=1}^{r-1} \sum_{j=k+2}^{v_{k}} s_{r-k, j}+\sum_{j=2}^{v_{0}} s_{r, j} \\
& =1+\sum_{k=1}^{r-1} s_{r-(k-1), k+1}+\sum_{j=2}^{v_{0}} s_{r, j} \\
& =s_{r, 1}+\sum_{j=2}^{v_{0}} s_{r, j}=S_{r} .
\end{aligned}
$$

(ii) Suppose there is only one nonzero term in the last row of $\Delta_{r}(n)$. Then

$$
\begin{aligned}
1+\sum_{k=0}^{r-1} \sum_{j=h_{k}+1}^{v_{k}} s_{r-k, j} & \leqq 1+\sum_{k=0}^{r-1} \sum_{j=k+2}^{v_{k}} s_{r-k, j} \\
& =1+\sum_{k=1}^{r-1} \sum_{j=k+2}^{v_{k}} s_{r-k, j} \\
& =1+\sum_{k=2}^{r-1} \sum_{j=k+2}^{v_{k}} s_{r-k, j}+\sum_{j=3}^{v_{1}} s_{r-1, j} \\
& =1+\sum_{k=2}^{r-1} s_{r-(k-1), k+1}+\sum_{j=3}^{v_{1}} s_{r-1, j} \\
& =s_{r-1,2}+\sum_{j=3}^{v_{1}} s_{r-1, j} \\
& =s_{r, 1} \equiv S_{r} .
\end{aligned}
$$


(B) We now show that the primary set $\left(h_{0}, h_{1}, \cdots, h_{r-1}\right)$ is an associate of $I$ (as defined by (5.1)) which we write as follows:

$$
I=1+\sum_{j=h_{0}+1}^{v_{0}} s_{r, j}+\sum_{j=h_{1}+1}^{v_{1}} s_{r-1, j}+\cdots+\sum_{j=h_{r-1+1}}^{v_{r-1}} s_{1, j} .
$$

We recall that $h_{k}$ by definition satisfies $1 \leqq h_{0}<h_{1}<\cdots<h_{r-1}$ and $h_{k} \leqq(n-3)-(r-(k+1)) 4=v_{k}$; also, that in determining the primary set associated with a given integer $g_{0}\left(1 \leqq g_{0} \leqq S_{r}\right)$ there is one and only one selection $j_{i}$ possible at each step (cf. (1), (2), (3) of $\S 4$ ). Thus, if we can show that

$$
\sum_{j=h_{0}+1}^{v_{0}} s_{r, j}<I \leqq \sum_{j=h_{0}}^{v_{0}} s_{r, j}
$$

then $j_{0}=h_{0}$. And, if we can show that if $j_{0}=h_{0}, j_{1}=h_{1}, \cdots, j_{i-1}$ $=h_{i-1}$ then

$$
\sum_{j=h_{i}+1}^{v_{i}} s_{r-i, j}<q_{i}^{\prime} \leqq \sum_{j=h_{i}}^{v_{i}} s_{r-i, j}
$$

where

$$
q_{i}^{\prime}=1+\sum_{j=h_{i}+1}^{v_{i}} s_{r-i, j}+\sum_{j=h_{i+1}+1}^{v_{i+1}} s_{r-(i+1), j}+\cdots+\sum_{j=h_{r-1+1}}^{v_{r-1}} s_{1, j}
$$

then $j_{i}=h_{i}(i=1, \cdots, r-1)$.

The left inequalities of (5.3) and (5.4) are obvious. The right inequalities of (5.3) and (5.4) will be true if we can show that

$$
\begin{array}{r}
1+\sum_{j=h_{i+1}+1}^{v_{i+1}} s_{r-(i+1), j}+\sum_{j=h_{i+2}+1}^{v_{i+2}} s_{r-(i+2), j}+\cdots+\sum_{j=h_{r-1+1}}^{v_{r-1}} s_{1, j} \\
\leqq s_{r-i}, h_{i},
\end{array}
$$

for $i \geqq 0$ (and then add $\sum_{j=h_{i}+1}^{j_{i}} s_{r-i, j}$ to both sides).

ProOF of (5.6). CASE I. Suppose $s_{r-i, h_{i}+1} \neq 0$. Then

$$
\begin{aligned}
& \sum_{j=h_{i+1}+1}^{v_{i+1}} s_{r-(i+1), j} \leqq \sum_{j=h_{i}+2}^{v_{i+1}} s_{r-(i+1), j}=s_{r-i, h_{i}+1} \\
& \sum_{j=h_{i+2}+1}^{v_{i+2}} s_{r-(i+2), j} \leqq \sum_{j=h_{i}+3}^{v_{i}+2} s_{r-(i+2), j}=s_{r-(i+1), h_{i}+2} \\
& \sum_{j=h_{r-1}+1}^{v_{r}-1} s_{1, j} \leqq \sum_{j=h_{i}+(r-i)}^{v_{r}-1} s_{1, j}=s_{2, h_{i}+r-(i+1)} .
\end{aligned}
$$


But by the lemma of $\S 3$,

$$
s_{r-i, h_{i}}=s_{r-i, h_{i}+1}+s_{r-(i+1), h_{i}+2}+\cdots+s_{2, h_{i}+r-(i+1)}+1 .
$$

Therefore (5.6) is true (in this case) for $i \geqq 0$.

CASE II. Suppose $s_{r-i, h_{i}+1}=0$. Then, using the ideas appearing in the proof of Case I, the left side of (5.6) is less than or equal to

$$
\begin{gathered}
1+\left(\sum_{j=h_{i}+2}^{v_{i}+1} s_{r-(i+1), j}\right)+s_{r-(i+1), h_{i}+2}+s_{r-(i+2), h_{i}+3}+\cdots+s_{2, h_{i}+r-(i+1)} \\
=\left(\sum_{j=h_{i}+2}^{v_{i+1}} s_{r-(i+1), j}\right)+s_{r-(i+1), h_{i}+1}=\sum_{j=h_{i}+1}^{v_{i}+1} s_{r-(i+1), j}=s_{r-i, h_{i}}
\end{gathered}
$$

for $i \geqq 0$.

6. The number of primary sets $\left(a_{1}, \cdots, a_{r}\right)$ (continued). In $\$ 4$ we have associated with each integer $g_{0}\left(1 \leqq g_{0} \leqq S_{r}\right)$ a primary set $\left(j_{0}, j_{1}, \cdots, j_{r-1}\right)$. In $\$ 5$ we have shown that each primary set $\left(h_{0}, h_{1}, \cdots, h_{r-1}\right)$ is the associate of an integer $I\left(1 \leqq I \leqq S_{r}\right)$. We show that this correspondence is $1-1$ reciprocal:

(i) Each integer $g_{0}\left(1 \leqq g_{0} \leqq S_{r}\right)$ has one and only one associated primary set $\left(j_{0}, j_{1}, \cdots, j_{r-1}\right)$ because in selecting the $j_{i}$ 's one and only one $j_{i}$ can be selected at each step (cf. $\S 4$ ).

(ii) Integers $g_{0}$ and $g_{1}\left(1 \leqq g_{0} \leqq S_{r}, 1 \leqq g_{1} \leqq S_{r}, g_{0} \neq g_{1}\right)$ cannot be associated with the same primary set $\left(j_{0}, j_{1}, \cdots, j_{r-1}\right)$. For in the contrary case $g_{0}$ and $g_{1}$ would each be equal to the right side of (4.11), and therefore to each other, which is impossible.

Thus there are $S_{r}$ primary sets of order $r$. If $r$ now varies over the range $1 \leqq r \leqq[n / 4]$, then there are as many primary sets of positive order as the sum of the terms of $\Delta_{m}(n)(m=[n / 4])$. Adjoining the primary set of order zero we have Theorem 1 as stated.

7. Proof of Theorem 2. Proof of (i). We recall that $\left(z_{1}, \cdots, z_{n}\right)$ is fixed. Let a primary set $\left(a_{1}, \cdots, a_{r}\right)$ (which may be of order zero) be given. Then with each $k(k \in\{1, \cdots . n\})(2.4)$ or (2.5) associates one and only one integer $p_{k}(=0$ or 1$)$ accordingly as $k$ belongs to $F$ (which may be null) or to $N$ (cf. (2.3)). Thus with each primary set is associated one and only one $n$-tuple $\left(p_{1}, \cdots, p_{n}\right)$. Applying $H^{-1}$ to $\left(p_{1}, \cdots, p_{n}\right)$ we obtain the unique lattice point associated with the primary set $\left(a_{1}, \cdots, a_{r}\right)$.

ProOF OF (ii). Let distinct primary sets

$$
\left(a_{1}, \cdots, a_{r}\right) \text { and }\left(a_{1}^{\prime}, \cdots, a_{s}^{\prime}\right)
$$


be given $\left(r \geqq s ;\left(a_{1}^{\prime}, \cdots, a_{s}^{\prime}\right)\right.$ may be the null set). Then (2.4) and (2.5) associate with (7.1)

$$
\left(p_{1}, \cdots, p_{n}\right) \text { and }\left(p_{1}^{\prime}, \cdots, p_{n}^{\prime}\right)
$$

respectively. Since the primary set (7.1) are distinct, there must be an $a_{i}$ such that $a_{i} \notin\left\{a_{1}^{\prime}, \cdots, a_{s}^{\prime}\right\}$. The $n$-tuplets (7.2) will be distinct since $p_{a_{i}} \neq p_{a_{i}}^{\prime}$. Therefore the lattice points associated with the primary sets (7.1) will be distinct.

City College, New York

\section{SUBGROUPS OF THE UNIMODULAR GROUP1}

IRVING REINER

Following the notation of [3], we let $\Gamma$ denote the proper unimodular group consisting of all $2 \times 2$ matrices with rational integral elements and determinant +1 . For $m$ a positive integer, define the principal congruence group $\Gamma(m)$ by

$$
\Gamma(m)=\{X \in \Gamma: X \equiv I(\bmod m)\},
$$

where $I$ denotes the identity matrix in $\Gamma$, and where congruence of matrices is interpreted as elementwise congruence.

For $p$ a prime, we know from [2] that $\Gamma(p)$ is a free group with a finite set $S$ of generators. If we define

$$
T_{m}=\left(\begin{array}{cc}
1 & m \\
0 & 1
\end{array}\right)
$$

then $S$ may be chosen to include $T_{p}$. For each fixed integer $s$, we may define a group $\Omega(p, s)$ consisting of all power products of the generators in $S$ for which the exponent sum for each generator is a multiple of $s$. In [3] it was shown that each $\Omega(p, s)$ is a normal subgroup of $\Gamma$ of finite index in $\Gamma$. Furthermore, if $s>1$ and $(s, p)=1$, it was proved that $\Omega(p, s)$ does not contain any principal congruence group.

Let $\Delta(m)$ denote the normal subgroup of $\Gamma$ which is generated by $T_{m}$. Obviously $\Delta(m) \subset \Gamma(m)$. Recently, Brenner [1] raised the following questions:
A. Does $\Delta(m)=\Gamma(m)$ for all $m$ ?

Received by the editors May 2, 1960.

1 This research was supported by the Office of Naval Research. 